\title{
Role of endoscopic ultrasound-guided fine-needle aspiration in evaluating mediastinal and intra-abdominal lymphadenopathies of unknown origin
}

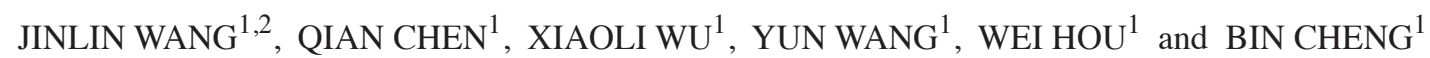 \\ Departments of ${ }^{1}$ Gastroenterology and Hepatology, and ${ }^{2}$ Oncology, Tongji Hospital, Tongji Medical College, \\ Huazhong University of Science and Technology, Wuhan, Hubei 430030, P.R. China
}

Received May 25, 2016; Accepted July 27, 2017

DOI: $10.3892 / \mathrm{ol} .2018 .8253$

\begin{abstract}
The aim of the present study was to evaluate the efficacy of endoscopic ultrasound-guided fine-needle aspiration (EUS-FNA) in diagnosing mediastinal and intra-abdominal lymphadenopathies. A total of 154 patients with mediastinal and intra-abdominal lymphadenopathies were included in this retrospective study between February 2010 and March 2015. Malignancy was suspected in the patients as a result of imaging findings and EUS-FNAs were performed to confirm the diagnoses. EUS and EUS-FNA data, as well as hospital medical records, were reviewed. The accuracy of EUS-FNA was $90.8 \%$ for diagnosing malignancy and $85.6 \%$ for diagnosing benign lymphadenopathy. In combination with flow cytometry (FCM), the accuracy of EUS-FNA to determine lymphoma was $94.2 \%$. Among the malignant lymphadenopathy cases, 80 were caused by metastasis, 19 by lymphoma and 1 by myeloid leukemia. In the 53 benign cases, EUS-FNA revealed a nonspecific inflammatory condition in 27 patients, tuberculosis in 21 patients and Castleman's disease in 5 patients. The factors revealed to be associated with malignant lymphadenopathy included the sex and age of patients, as well as the location and size of the enlarged lymph node. In particular, celiac axis lymphadenopathy was associated with malignancy (23.0\% of cases of malignancy, vs. $3.8 \%$ of benign lymphadenopathy). EUS-FNA results additionally suggested that the malignant lymph nodes observed in celiac axis were more likely to result from lymphoma $(42.1 \%$; $8 / 19$ cases) than metastasis $(18.8 \%$; $15 / 80$ cases; $\mathrm{P}=0.039)$. By contrast, malignant lymph nodes observed in the mediastinum were more likely to be caused by metastasis $(47.5 \%$; $38 / 80$ cases) than
\end{abstract}

Correspondence to: Professor Bin Cheng, Department of Gastroenterology and Hepatology, Tongji Hospital, Tongji Medical College, Huazhong University of Science and Technology, 1095 Jie Fang Avenue, Wuhan, Hubei 430030, P.R. China

E-mail: b.cheng@tjh.tjmu.edu.cn

Key words: endoscopic ultrasound-guided fine-needle aspiration, lymphadenopathy, metastasis, lymphoma, flow cytometry lymphoma $(10.5 \% ; 2 / 19$ cases; $\mathrm{P}=0.004)$. The results of the present study suggested that EUS-FNA is accurate for differentiating between malignancy and benign lymphadenopathy. Therefore, EUS-FNA in combination with FCM analysis, as a minimally invasive and highly sensitive tool, should be routinely performed for the identification of lymphoma. Additionally, examining the enlarged celiac axis lymph nodes of elderly males, who exhibit an increased risk of malignancy, may be beneficial.

\section{Introduction}

Mediastinal and intra-abdominal lymphadenopathies may present with numerous benign or malignant conditions. Benign lymph node enlargement may occur as a response to certain infections, including tuberculosis and fungal infection, as well as several less common diseases, including Castleman's disease and Wegener's granulomatosis. Malignant lymphadenopathy may occur in primary lymphatic diseases such as Hodgkin's lymphoma (HL) and non-Hodgkin's lymphoma (NHL), as well as in other types of malignancy that have metastasized to regional lymph nodes, including lung, esophageal, breast and pancreatic carcinomas $(1,2)$.

Advanced imaging methods, including computed tomography (CT), magnetic resonance imaging and positron emission tomography-computed tomography (PET-CT), serve important roles in identifying enlarged lymph nodes during the staging workup for lymphoproliferative disorders; however, they may be limited in their ability to differentiate between inflammatory/reactive processes and malignancy (3-5). Thus, tissue obtained percutaneously or surgically is critical to providing a pathological diagnosis, including insight into the possible disease origin. Laparoscopic lymph node biopsy and image-guided needle biopsy (IGNB) have been applied to obtain tissue from enlarged lymph nodes; however, these are invasive and expensive procedures. Endoscopic ultrasound (EUS) has emerged as a novel imaging modality $(3,5)$. A fine-needle aspiration (FNA) biopsy coupled with EUS allows for the sampling of the target lesion with ultrasound guidance in real time, providing minimally invasive access to the lymph nodes within the abdomen and mediastinum $(6,7)$. Compared with IGNB, EUS-FNA exhibits increased 
accuracy for the sampling of anatomically challenging sites, including those close to major blood vessels, which is useful for avoiding injury to the adjacent tissue (7). In addition, EUS-FNA allows access to deep-seated lymph nodes and the sampling of small lesions $(<25 \mathrm{~mm})$, which overcomes the limitations of sampling through other techniques (8). Previous studies have demonstrated that, for diagnosing mediastinal and intra-abdominal lymphadenopathies, EUS-FNA exhibits a sensitivity of 79-98.3\%, a specificity of 98-100\%, a positive predictive value (PPV) of $98-100 \%$, a negative predictive value (NPV) of $62-98.4 \%$ and an accuracy of $84-99.4 \%(9,10)$.

The aim of the present study was to evaluate the efficacy and safety of EUS-FNA for the diagnosis of mediastinal and intra-abdominal lymphadenopathies of unknown origin.

\section{Patients and methods}

Patients. The present retrospective study included 154 patients with mediastinal and intra-abdominal lymphadenopathies that were suspected to be malignant based on imaging data. All patients underwent EUS-FNA at Tongji Hospital (Wuhan, China) between February 2010 and March 2015. The present study was approved by The Ethics Committee of Tongji Hospital and informed consent was obtained from each patient or their legally authorized representative. The following data were collected from the hospital medical record system: Age, sex, EUS findings, EUS-FNA findings, procedure-associated complications and follow-up results.

EUS-FNA procedure. EUS-FNA was performed under deep sedation according to the principles of "monitored anesthesia care' (11). The patients were anaesthetized with the intravenous administration of Propofol $(2 \mathrm{ml} / \mathrm{kg}$ ). All patients received oxygen during the procedure and their blood pressure and heart rate were monitored.

The EUS-FNA examinations were conducted using an Olympus linear echoendoscope (GF-UCT 240, GF-UCT 260; Olympus Corporation, Tokyo, Japan) platform with the Aloka Processor Alpha-5 (Hitachi, Ltd., Tokyo, Japan). An endosonographer with $>5$ years of EUS experience, performing $>150$ EUS-FNAs per year, performed EUS-FNA in the present study. A 22-gauge EchoTip Ultra needle (Cook Endoscopy; Cook Medical Inc., Bloomington, IN, USA) was advanced into lesions with real-time EUS visualization. The endosonographer maneuvered the needle back and forth 20 times within the lesion, applying minimal negative pressure by pulling the needle stylet slowly and continuously. If no specimen was obtained, continuous suction was applied with a 5-10 $\mathrm{ml}$ syringe in order to obtain a specimen. Samples were then prepared for histological and cytological examinations. The specimens were expelled from a needle in three steps: i) pushing the stylet into the needle; ii) flushing the needle with $0.1 \mathrm{ml}$ saline; and iii) filling the needle with $2 \mathrm{ml}$ of air. The core tissue was put into the container filled with formaldehyde for histological examination. The fragment of the specimen was put on the glass slide, and another glass slide was used to smear for cytological examinations. In suspected cases of lymphoma, additional samples were obtained for flow cytometry (FCM).
FCM. FCM was performed on a FACScan analyzer (BD Biosciences, Franklin Lakes, NJ, USA) using standard procedures $(12,13)$. The cells were stained with selected monoclonal antibodies as required $(30 \mathrm{~min}$ at room temperature), from the following: Immunoglobulin $\kappa$ and $\lambda$ light chains (cat no. 560950, 1:5; cat no. 562054, 1:5; BD Biosciences, Franklin Lakes, NJ, USA), cluster of differentiation CD2 (cat no. 555327; 1:5; BD Biosciences), CD3 (cat no. 552127; 1:5; BD Biosciences), CD4 (cat no. 561842; 1:20; BD Biosciences), CD5 (cat no. 555353; 1:5; BD Biosciences), CD8 (cat no. 557085; 1:5; BD Biosciences), CD10 (cat no. 561002; 1:5; BD Biosciences), CDllc (cat no. 560999; 1:5; BD Biosciences), CD19 (cat no. 555413; 1:5; BD Biosciences), CD20 (cat no. 560961; 1:5; BD Biosciences), CD23 (cat no. 561146; 1:20; BD Biosciences), CD25(cat no. 560990; 1:5; BD Biosciences), CD38 (cat no. 555462; 1:5; BD Biosciences), FMC-7 (cat no. 340919; 1:5; BD Biosciences)-a normal B-cell antigen expressed on mature human $B$ cell and it is used in during immunophenotypic analysis and differential diagnosis of lymphomas and leukemias (14), major histocompatibility complex (cat no. ab63567; 1:10; Abcam, Cambridge, MA, USA), class II-DR (HLA-DR) (cat no. 560896; 1:5; BD Biosciences), CD30 (cat no. 555829; 1:5; BD Biosciences), CD45 (cat no. 340953; 1:5; BD Biosciences), CD103 (cat no. 563883; 1:20; BD Biosciences), CDl38 (cat no. 564605; 1:20; BD Biosciences), ki-67 (cat no. 612472; 1:5; BD Biosciences), Bcl-2 (cat no. 562679; 1:20; BD Biosciences), CDl6 (cat no. 565421; 1:20; BD Biosciences), CD56 (cat no. 565140; 1:20; BD Biosciences), CD57 (cat no. 560845; 1:20; BD Biosciences), CD94 (cat no. 562361; 1:20; BD Biosciences), CD161 (cat no. 551138; 1:5; BD Biosciences), CD158a/h (cat no. 564319; 1:20; BD Biosciences), CD158b (cat no. 566053; 1:20; BD Biosciences), CDl58e (cat no. 564103; 1:20; BD Biosciences), T-cell receptor (TCR)ab (cat no. 563826; 1:20; BD Biosciences), and TCRrd (cat no. 564157; 1:5; BD Biosciences).

Follow up. The final diagnosis was typically based on the pathological report obtained from surgery. However, if the patient did not undergo surgery, a 9 month follow-up was performed (15). Final diagnosis was classified as either: i) Malignant lymphadenopathy: EUS-FNA cytology and/or pathology results revealed malignant cells that were confirmed by specific immunohistochemical stains or supported with FCM results; or ii) benign lymphadenopathy: Absence of typical malignant cell morphology with no further signs of malignancy, i.e. the lesions exhibited regression or no further progression at follow-up, as previously described $(9,16)$.

Statistical analysis. EUS-FNA-associated parameters, including the sensitivity, specificity, PPV, NPV and accuracy were evaluated by comparing EUS-FNA results with the final diagnoses. Continuous variables are reported as the mean \pm standard deviation and categorical variables are reported as percentages. Student's t-tests were used to compare continuous variables and categorical variables were compared using $\chi^{2}$-tests. A two-tailed P-value of $<0.05$ was considered to indicate a statistically significant difference. All data were processed and analyzed using SPSS (version 19; IBM Corp., Armonk, NY, USA). 
Table I. Patient and lesion characteristics of malignant and benign lymphadenopathy.

\begin{tabular}{|c|c|c|c|}
\hline Patient characteristics & Malignant lymphadenopathy $(n=100)$ & Benign lymphadenopathy $(n=53)$ & P-value \\
\hline Sex, $n$ & & & $0.0110^{\mathrm{a}}$ \\
\hline Male & 75 & 29 & \\
\hline Female & 25 & 24 & \\
\hline Age, years $^{\mathrm{b}}$ & $55 \pm 11.9$ & $43 \pm 16.8$ & $0.0001^{\mathrm{a}}$ \\
\hline \multicolumn{4}{|l|}{ Lesion location $^{c}$} \\
\hline Peripancreas & $32(32.0)$ & $21(39.6)$ & 0.3750 \\
\hline Around the celiac axis & $23(23.0)$ & $2(3.8)$ & $0.0020^{\mathrm{a}}$ \\
\hline Hepatic portal & $5(5.0)$ & $8(15.1)$ & 0.0630 \\
\hline Mediastinum & $40(40.0)$ & $22(41.5)$ & 0.8640 \\
\hline \multicolumn{4}{|c|}{ Endoscopic ultrasound characteristics } \\
\hline Size, $\mathrm{mm}^{\mathrm{b}}$ & $33.9 \pm 15.8$ & $24.8 \pm 13.8$ & $0.0110^{\mathrm{a}}$ \\
\hline Sharp margin $^{c}$ & $85(85.0)$ & $40(75.5)$ & 0.1470 \\
\hline Fusion $^{c}$ & $23(23.0)$ & $9(17.0)$ & 0.3840 \\
\hline Echogenic focus $^{c}$ & $25(25.0)$ & $15(28.3)$ & 0.6580 \\
\hline Homogeneous echogenicity $^{c}$ & $46(46.0)$ & $22(41.5)$ & 0.5950 \\
\hline Malignant echo-features ${ }^{c, d}$ & $40(75.5)$ & $81(81.0)$ & 0.1530 \\
\hline
\end{tabular}

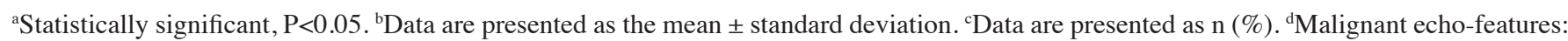
Hypoechoic, sharp margin, rounded and size $>10 \mathrm{~mm}$.

\section{Results}

Final diagnoses. A total of 154 patients (105 male, 49 female) were included in the present study. The mean patient age was 49.1 years (22-68 years). The success rate for obtaining pathological material by EUS-FNA was $87.0 \%(134 / 154)$ and adequate cytology samples were obtained in all cases. The mean number of needle passes was 2.9 (range, 1-6). For the 52 patients suspected to be presenting with lymphoma, the EUS-FNA samples were evaluated by cytology, pathology and FCM studies. Overall, a definitive diagnosis was obtained for 153 of the 154 patients (including 31 from surgical pathology and 122 from follow-up). A total of 100 patients were determined to exhibit malignant lymphadenopathy, including 80 cases of metastasis, 19 cases of lymphoma and 1 case of myeloid leukemia. The remaining 53 cases were determined to be benign, including 27 cases of a non-specific inflammatory condition, 21 cases of tuberculosis and 5 cases of Castleman's disease.

Patient and lesion characteristics. As presented in Table I, the incidence of malignant lymphadenopathy in the present study cohort was significantly increased in males compared with females $(\mathrm{P}=0.011)$. The observed mean age for patients with malignancy was $55 \pm 11.9$ years compared with $43 \pm 16.8$ years for the benign lymphadenopathy $(\mathrm{P}=0.0001)$. Other factors associated with malignancy included the location and size of the enlarged lymph node. The mean size of a malignant lymph node was $33.9 \pm 15.8 \mathrm{~mm}$, compared with $24.8 \pm 13.8 \mathrm{~mm}$ for a benign lymph node $(\mathrm{P}=0.011)$. In particular, the celiac axis lymphadenopathy was a clear indicator of malignancy $(23.0 \%$ in malignancy vs. $3.8 \%$ in benign lymphadenopathy; $\mathrm{P}=0.039$ ). However, the echo-features of lymph nodes as assessed during
EUS were not significantly different between malignant and benign cases $(\mathrm{P}=0.153)$; therefore, this may not be useful in diagnosing malignancy.

As presented in Table II, it was demonstrated thatEUS-FNA may be useful in assessing lymphadenopathy resulting from metastasis and lymphoma in the celiac axis, mediastinum, peripancreatic and hepatic portal areas. Malignant lymph nodes observed in the celiac axis were more likely to have resulted from lymphoma $(42.1 \%$; $8 / 19$ cases) than metastasis (18.8\%; 15/80 cases; $\mathrm{P}=0.039)$. In contrast, malignant lymph nodes observed in the mediastinum were more likely to have been caused by metastasis $(47.5 \%$; 38/80 cases) than lymphoma $(10.5 \%$; $2 / 19$ cases; $\mathrm{P}=0.004)$. EUS-FNA data revealed that the mean size of metastatic lymph nodes was $35.6 \pm 16.4 \mathrm{~mm}$, whereas it was $27.2 \pm 11.2 \mathrm{~mm}$ for lymphoma $(\mathrm{P}=0.011)$. Furthermore, lesion fusion was observed in $47.4 \%$ of lymphoma cases and $17.5 \%$ of metastasis cases $(\mathrm{P}=0.013)$. Homogeneous echogenicity was observed in $40.0 \%$ of the cases of metastasis and $73.7 \%$ of the cases of lymphoma $(\mathrm{P}=0.011)$. Thus, these results suggested a potential role for EUS-FNA in distinguishing cases of lymphoma from metastasis in the celiac axis and mediastinum.

Efficacy of EUS-FNA for identifying malignant lymphadenopathy. Among 100 patients diagnosed with malignant lymphadenopathy, the sensitivity, specificity, PPV, NPV and accuracy of EUS-FNA for malignant lymphadenopathy were $86.0,100.0,100.0,79.1$ and $90.8 \%$, respectively (Table III).

The predominant cause for lymphadenopathy was metastasis $(52.3 \%$; 80/154). The application of EUS-FNA generated a correct diagnosis for 70 patients, including squamous cell carcinoma $(n=28)$, adenocarcinoma $(n=32)$, neuroendocrine 
Table II. Patient and lesion characteristics of metastasis and lymphoma.

\begin{tabular}{|c|c|c|c|}
\hline Patient characteristics & Metastasis $(\mathrm{n}=80)$ & Lymphoma (n=19) & P-value \\
\hline Sex, $n$ & & & 0.2420 \\
\hline Male & 62 & 12 & \\
\hline Female & 18 & 7 & \\
\hline Age, years $^{\mathrm{a}}$ & $54.9 \pm 11.9$ & $55.4 \pm 12.8$ & 0.8950 \\
\hline \multicolumn{4}{|l|}{ Lesion location ${ }^{\mathrm{b}}$} \\
\hline Peripancreas & $23(28.8)$ & $9(47.4)$ & 0.2800 \\
\hline Around the celiac axis & $15(18.8)$ & $8(42.1)$ & $0.0390^{\mathrm{c}}$ \\
\hline Hepatic portal & $5(6.3)$ & $0(0.0)$ & 0.5800 \\
\hline Mediastinum & $38(47.5)$ & $2(10.5)$ & $0.0040^{\mathrm{c}}$ \\
\hline \multicolumn{4}{|c|}{ Endoscopic ultrasound characteristics } \\
\hline Size, $\mathrm{mm}^{\mathrm{a}}$ & $35.6 \pm 16.4$ & $27.2 \pm 11.2$ & $0.0110^{\mathrm{c}}$ \\
\hline Sharp margin $^{\mathrm{b}}$ & $66(82.5)$ & $19(100.0)$ & 0.0650 \\
\hline Fusion $^{\mathrm{b}}$ & $14(17.5)$ & $9(47.4)$ & $0.0130^{\mathrm{c}}$ \\
\hline Echogenic focus $^{b}$ & $24(30.0)$ & $1(5.3)$ & $0.0370^{c}$ \\
\hline Homogeneous echogenicity ${ }^{\mathrm{b}}$ & $32(40.0)$ & $14(73.7)$ & $0.0110^{\mathrm{c}}$ \\
\hline
\end{tabular}

${ }^{\mathrm{a}}$ Data are presented as the mean \pm standard deviation. ${ }^{\mathrm{b}}$ Data are presented as $\mathrm{n}(\%)$. ${ }^{\mathrm{c}}$ Statistically significant, $\mathrm{P}<0.05$.

Table III. Efficacy of endoscopic ultrasound-guided fine-needle aspiration.

\begin{tabular}{|c|c|c|c|c|c|}
\hline \multirow[b]{2}{*}{ Condition } & \multicolumn{5}{|c|}{ Metric, $\%$} \\
\hline & Sensitivity & Specificity & PPV & NPV & Accuracy \\
\hline Malignant lymphadenopathy & 86.0 & 100.0 & 100.0 & 79.1 & 90.8 \\
\hline Metastasis & 87.5 & 100.0 & 100.0 & 87.9 & 93.5 \\
\hline Lymphoma & 84.2 & 100.0 & 100.0 & 91.7 & 94.2 \\
\hline Benign lymphadenopathy & 58.5 & 100.0 & 100.0 & 81.9 & 85.6 \\
\hline Tuberculosis & 38.1 & 100.0 & 100.0 & 91.7 & 92.2 \\
\hline Nonspecific reactive lymphadenopathy & 100.0 & 77.7 & 54.2 & 100.0 & 82.4 \\
\hline
\end{tabular}

PPV, positive predictive value; NPV, negative predictive value.

tumor $(n=7)$ and undifferentiated carcinoma $(n=3)$. Among them, 30 patients had a history of tumors and the recurrence of malignancy was confirmed by EUS-FNA. Notably, the remaining 40 patients had no history of malignancy and no primary lesion had been detected prior to EUS-FNA. Thus, additional examination, including PET-CT, was applied to investigate the tumor origin following EUS-FNA; primary lesions were successfully identified in 35/40 patients. The sensitivity, specificity, PPV, NPV and accuracy of EUS-FNA for metastasis were $87.5,100.0,100.0,87.9$ and $93.5 \%$, respectively.

Of the 52 patients suspected to be presenting with lymphoma, 19 patients were confirmed, including 10 patients with diffuse large B-cell lymphomas (DLBCL), 2 patients with HL, 1 patient with T-cell lymphoma and 7 patients with B-cell NHL (B-NHL) without further subclassifications.

The cytological findings included 9 true-positive cases and 3 false-positive cases. Benign lymphadenopathy due to nonspecific inflammatory reaction was the final diagnosis in the 3 false-positive cases, which was determined by follow-up. The sensitivity, specificity, PPV, NPV and accuracy of EUS-FNA combined with cytology for lymphoma were 47.4, 90.9, 75.0, 75.0 and 75.0\%, respectively (Table IV).

Pathology via EUS-FNA provided adequate tissue samples for $84.2 \%$ of patients. Notably, the pathology analysis was not sufficient to diagnose 16 cases with malignant lesions, although there was no false-positive result. Only 3 patients were diagnosed correctly ( 2 cases with DLBCL and 1 case without definite subclassification). The sensitivity, specificity, PPV, NPV and accuracy of EUS-FNA combined with pathology for lymphoma were 15.8, 100.0, 100.0, 67.3 and $69.2 \%$, respectively.

The combined analysis of EUS-FNA and FCM contributed to the correct diagnosis of 16 patients with B-NHL through detecting light-chain restriction expression (Fig. 1). A total of 8 lymphomas were further subtyped as DLBCL due to 
Table IV. Efficacy of EUS-FNA in the identification of lymphoma.

Final diagnoses, $\mathrm{n}$

\begin{tabular}{|c|c|c|c|c|c|c|c|c|}
\hline Method & $\mathrm{n}$ & Lymphoma & Non-lymphoma & Sensitivity, \% & Specificity, \% & PPV, \% & NPV, $\%$ & Accuracy, $\%$ \\
\hline $\begin{array}{l}\text { EUS-FNA with } \\
\text { flow cytometry }\end{array}$ & 52 & & & 84.2 & 100.0 & 100.0 & 91.7 & 94.2 \\
\hline Lymphoma & & 16 & 0 & & & & & \\
\hline Non-lymphoma & & 3 & 33 & & & & & \\
\hline $\begin{array}{l}\text { EUS-FNA with } \\
\text { cytology }\end{array}$ & 52 & & & 47.4 & 90.9 & 75.0 & 75.0 & 75.0 \\
\hline Lymphoma & & 9 & 3 & & & & & \\
\hline Non-lymphoma & & 10 & 30 & & & & & \\
\hline $\begin{array}{l}\text { EUS-FNA with } \\
\text { pathology }\end{array}$ & 52 & & & 15.8 & 100.0 & 100.0 & 67.3 & 69.2 \\
\hline Lymphoma & & 3 & 0 & & & & & \\
\hline Non-lymphoma & & 16 & 33 & & & & & \\
\hline
\end{tabular}

EUS-FNA, endoscopic ultrasound-guided fine-needle aspiration; PPV, positive predictive value; NPV, negative predictive value.

the detection of CD3, CD5, CD10, Bcl-2 and CD20 expression by FCM (Fig. 1). There were no false-positive cases. The combined EUS-FNA and FCM method was not able to determine 2 cases of HL or 1 case of T-cell lymphoma, which were ultimately diagnosed with excisional biopsies. The sensitivity, specificity, PPV, NPV and accuracy of EUS-FNA combined with FCM assessing lymphoma were 84.2, 100.0, 100.0, 91.7 and $94.2 \%$, respectively. Thus, the efficacy of the combined analysis is markedly higher than cytology $(\mathrm{P}<0.001)$ or pathology $(\mathrm{P}=0.001)$ alone (data not shown).

In one atypical case, a 16-year-old male experienced intermittent abdominal pain for 6 months in the absence of fever. Laboratory investigations were performed on peripheral blood counts, whereas a CT scan revealed retroperitoneal lymphadenopathy. EUS-FNA was therefore recommended to evaluate the enlarged lymph nodes (Fig. 2). Although cytology supported the diagnosis of NHL based on immature lymphocyte proliferation and atypical small, round cells, a definitive conclusion could not be drawn. A subsequent FCM investigation highlighted the presence of abnormal cells with a characteristic phenotype of malignant myeloid origin, which expressed CD117, CD56, CD34, CD13, CD33 and HLA-DR, partially expressed CD19, cMPO, CD11c and CD15, and did not express CD3, CD4, CD8, CD5, CD20, CD23,, , $\lambda$, cCD79a, cCD3 or CD16 (Fig. 2). The patient underwent a bone marrow biopsy and the diagnosis of acute myeloid leukemia was confirmed.

Efficacy of EUS-FNA for benign lymphadenopathy. For the patients with benign lymphadenopathy, the median follow-up period was 10.2 months (range 9-48 months). The sensitivity, specificity, PPV, NPV and accuracy of EUS-FNA for benign lymphadenopathy were $58.5,100.0,100.0,81.9$ and $85.6 \%$, respectively. Tuberculosis $(n=21)$ was the most common etiology, with the exception of a nonspecific inflammatory condition $(n=27)$. The diagnosis of tuberculosis in 8 patients
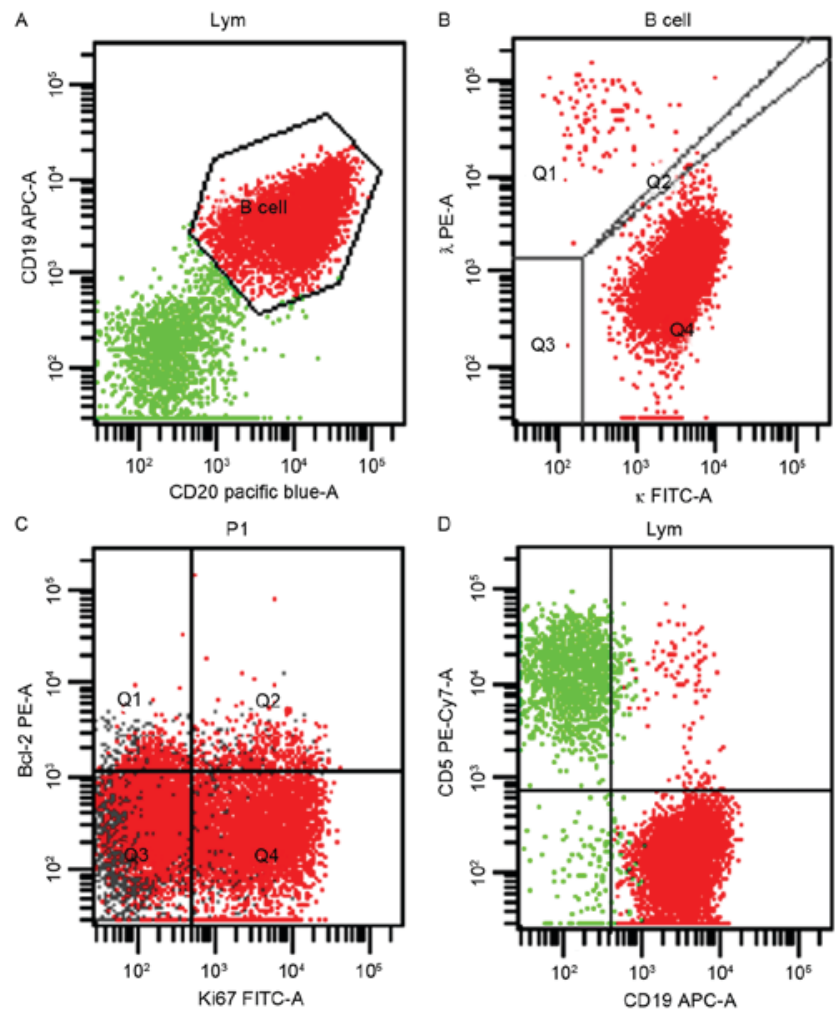

Figure 1. Diffuse large B-cell lymphoma diagnosed using endoscopic ultrasound-guided fine-needle aspiration combined with FCM. (A) FCM scatter plot with CD19/CD20 identified a group of B cells. (B) The group of $B$ cells exhibited restricted $\kappa$ light-chain expression. (C) FCM identified a population of Bcl-2/Ki-67+ cells. (D) FCM scatter plot with $\mathrm{CD} / \mathrm{CD} 19$ identified a population of $\mathrm{CD}^{-} / \mathrm{CD} 19^{+}$cells. FCM, flow cytometry; $\mathrm{CD}$, cluster of differentiation; APC, allophycocyanin; PE, phycoerythrin; FITC, fluorescein isothiocyanate; lym, lymphocyte; P1, target cells.

depended on the histological identification of granulomatous lymphadenitis without acid-fast bacilli (Fig. 3). The 

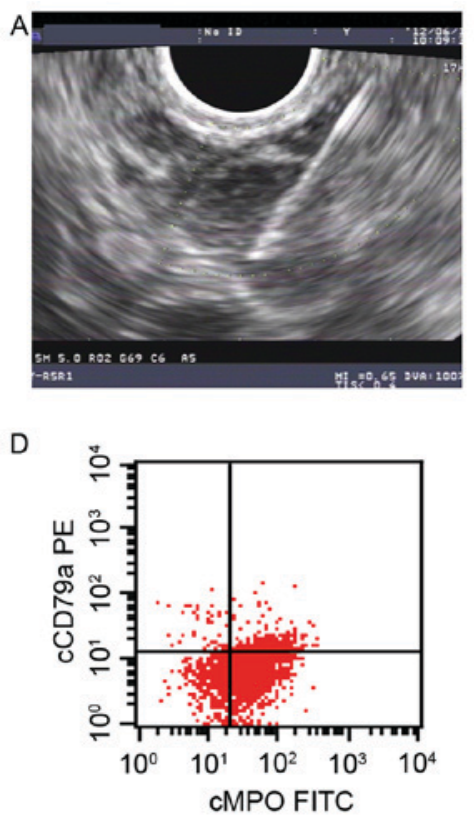
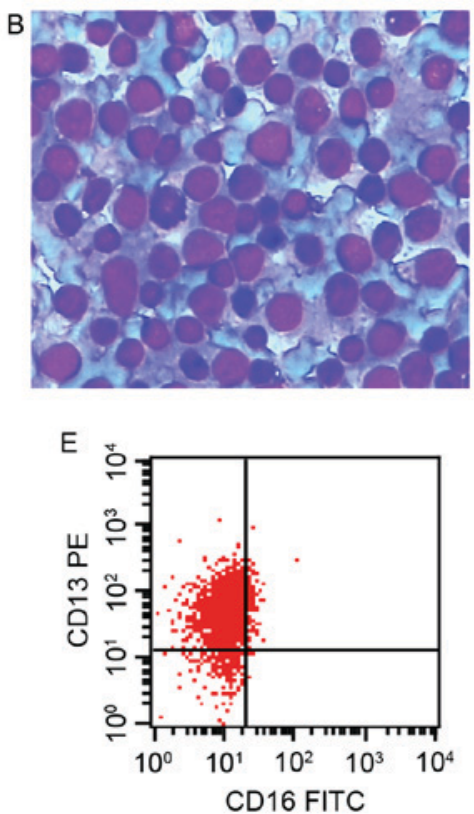
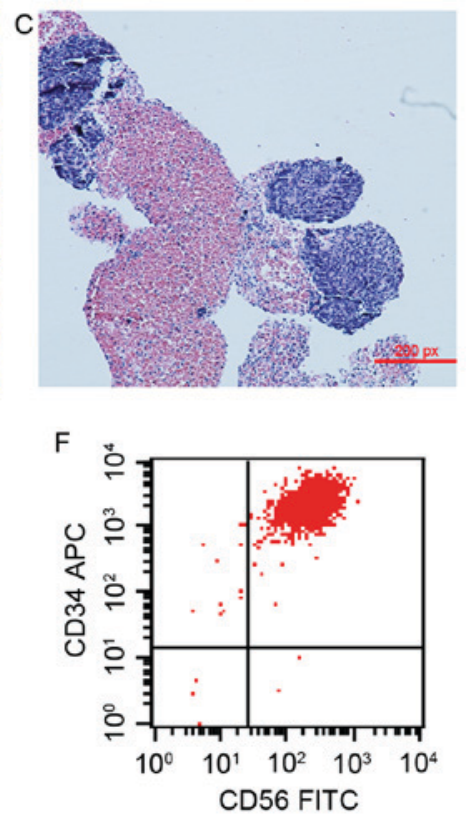

Figure 2. A case of myeloid leukemia accompanied by extramedullary infiltration, diagnosed using EUS-FNA combined with FCM. (A) EUS image of retroperitoneal lymphadenopathy; EUS-FNA was performed using a 22-gauge needle. (B) Cytology identified the active proliferation of immature lymphocytes, suspected to be non-Hodgkin's lymphoma (Liu's stain; magnification, x400). (C) Pathology revealed small, round cells (hematoxylin and eosin stain; magnification, x100). (D) A FCM scatter plot with cCD79a/cMPO revealed cCD79a-cMPO ${ }^{+}$cells, which suggested that the abnormal cells were derived from the myeloid hematopoietic cells. (E) A FCM scatter plot for CD13/CD16 revealed CD13 ${ }^{+} / \mathrm{CD} 16^{-}$cells, which suggested that the abnormal cells were derived from granulocytes. (F) FCM scatter plot for CD34/CD56 revealed $\mathrm{CD} 13^{+} / \mathrm{CD} 16^{+}$cells, which suggested the abnormal cells were precursors. APC, allophycocyanin; PE, phycoerythrin; FITC, fluorescein isothiocyanate; EUS-FNA, endoscopic ultrasound-guided fine-needle aspiration; CD, cluster of differentiation; FCM, flow cytometry; cMPO, myeloperoxidase on the cell membrane.
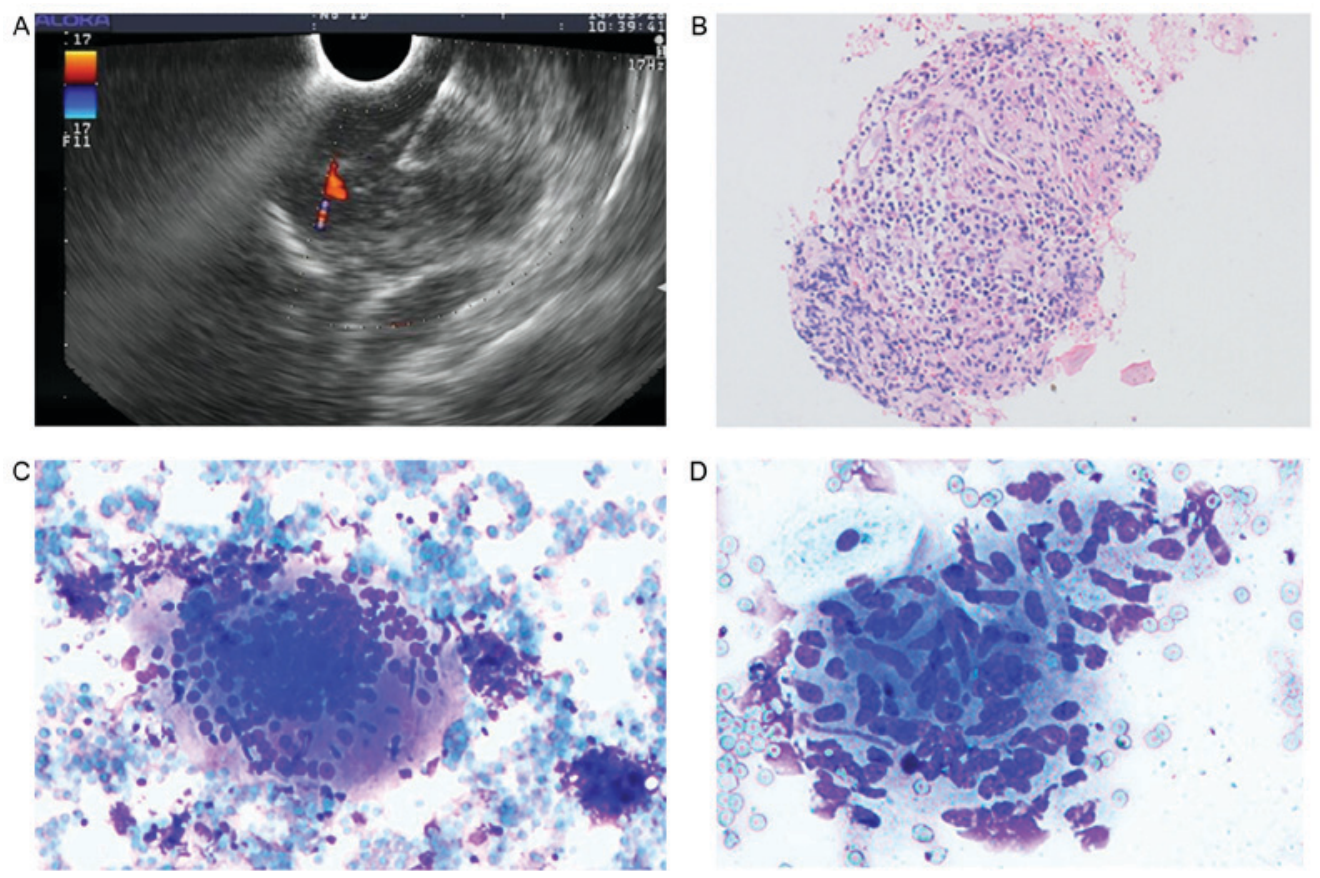

Figure 3. A case of tuberculosis diagnosed by EUS-FNA. (A) EUS images of retroperitoneal lymphadenopathy, presenting as two masses measuring 3.0x1.8 and $1.6 \times 0.8 \mathrm{~cm}$ in the mediastinum, with a ill-defined border, heterogeneous echogenicity, echogenic focus and lesion fusion; EUS-FNA was performed with a 22-gauge needle with 5-ml suction pressure. (B) Pathology identified a granuloma (hematoxylin and eosin stain; magnification, x200). Cytology revealed (C) Langerhans giant cells (Liu's stain; magnification, x100) and (D) epithelioid cells (Liu's stain; magnification, x200). EUS-FNA, endoscopic ultrasound-guided fine-needle aspiration.

sensitivity, specificity, PPV, NPV and accuracy of EUS-FNA assessing tuberculosis were $38.1,100.0,100.0,91.7$ and $92.2 \%$, respectively.
Complications. None of the patients experienced any severe peri- or post-procedural complications, as previously defined (17), including hemorrhage or infection. 


\section{Discussion}

EUS-FNA may influence patient management, as a doctor will give patients different advice according to the results of EUS-FNA. For example if the results show benign disease, the doctor may suggest follow-up or conservative treatment. However, if the results indicate malignant disease, the doctor may suggest aggressive treatment. The diagnostic ability of EUS-FNA in regards to lymphadenopathies is well established $(3,18)$. The present retrospective study of 154 patients is one of the largest studies conducted on the topic, to the best of our knowledge. The data collected suggest that EUS-FNA is an efficient and safe method for assessing mediastinal and intra-abdominal lymphadenopathies of unknown origin. In the present study, $65.4 \%$ of the enlarged lymph nodes were determined to be malignant. Elderly and male patients were identified to possess an increased risk of malignant disease and, in addition, lymphadenopathy in the region of the celiac axis was more likely to be caused by malignant disease. Echo-features are an important assessment during EUS; however, criteria for their assessment to predict malignant lymph node invasion have yet to be established. Although Catalano et al (19) suggested that a number of echo-features, including hypoechoic, sharp borders, rounded contour and size $>10 \mathrm{~mm}$, were associated with malignancy, the conclusions were not supported by similar studies $(20,21)$. The results of the present study did not indicate an association between echo-features and malignant lymphadenopathy; however, the size of malignant lymph nodes were significantly increased compared with benign lymph nodes.

Taking into consideration the anatomical and technical challenges associated with accessing the intra-abdomen or mediastinum, the application of EUS-FNA may benefit the patients with lymph node metastasis or lymphoma more than others (15). In the present study, an increased proportion of the enlarged lymph nodes located in the mediastinum were metastatic lymph nodes, whereas enlarged lymph nodes caused by lymphoma were predominantly located around the celiac axis. In addition, metastatic lymph nodes were larger and exhibited echogenic focus, whereas enlarged lymph nodes caused by lymphoma were characterized by lesion fusion and homogeneous echogenicity. These results may provide insight into conducting a differential diagnosis between metastatic lymph nodes and lymphoma in the clinical work-up.

Patients with benign lymphadenopathies do not require intensive treatment; typically, routine patient follow-up is sufficient, depending on the nature of the pathology. By contrast, the patients diagnosed with malignancy require the determination of tumor origin and are subsequently referred for surgical resection when possible, chemo/radiation therapy and/or appropriate palliative care $(4,20)$. PET-CT is considered to be a valuable predictive and prognostic tool for patients with malignancy; however, its sensitivity and NPV are poor (22), and the biopsy result remains the key in guiding the clinician's management choice (17). Consistent with a previous study by Dewitt et al (23), the present study demonstrated that EUS-FNA improved the detection of the postoperative recurrence of malignancy, potentially allowing for cancer staging to be performed prior to surgery.
FCM technology allows for the assessment of abnormal cell phenotypes and thus improves the sensitivity, specificity and accuracy of EUS-FNA in diagnosing primary or recurrent lymphoma $(1,24,25)$. In the present study, FCM was performed on samples from 52 patients with suspicions of lymphoma. The sensitivity, specificity, PPV, NPV and accuracy of EUS-FNA combined with FCM for lymphoma were $84.2,100,100,91.7$ and $94.2 \%$, respectively. The overall efficacy was markedly increased compared with pathological and cytological analyses.

Another advantage of the combined EUS-FNA and FCM analysis is that it typically provides a sufficient specimen to allow further lymphoma subtyping, so that patients may be given the appropriate therapy (26). Consistent with a previous study on subtyping B-NHL (3), the present study demonstrated that EUS-FNA in combination with FCM markedly improved the diagnosis efficiency in cases of deep-seated lymphoma; $84.2 \%$ of the patients with lymphoma were diagnosed with B-NHL, and this method correctly subtyped $50 \%$ of B-NHL cases.

There are several limitations for EUS-FNA as an independent tool to provide a definitive diagnosis of lymphoma. Firstly, the diagnosis of HL requires integration of cytomorphology, immunophenotypical and clinical features, and thus may continue to require open biopsies for diagnosis and classification (12). In the present study, 2 cases of HL were ultimately diagnosed using excisional biopsy. Secondly, the diagnosis of T cell lymphomas could not be performed with FCM due to the lack of specific antibodies for detection $(12,13)$. T cell lymphoma rarely occurs in the gastrointestinal tract, mediastinum or retroperitoneum (27); the present study identified only 1 case of $\mathrm{T}$ cell lymphoma, which was eventually determined by open biopsy. Third, given the reliance on adequate material for FCM analysis, the quality of sampling requires further improvement. A total of $\geq 3$ passes from several angles of the lymph nodes may be necessary to obtain adequate samples (12). In addition, avoiding necrotic tissue and blood contamination during sampling is critical.

In the present study, a rare case of myeloid leukemia with extramedullary leukemic cell infiltration was identified. The patient presented with abdominal pain in the absence of typical myeloid leukemia symptoms, including fever, anemia, bleeding and infection. Although malignant myeloid precursor cells have been demonstrated to infiltrate and proliferate in multiple organ systems (28), limited methods are available to confirm diagnosis. To the best of our knowledge, the present study is the first to demonstrate that EUS-FNA combined with FCM was able to determine the characteristic phenotype of malignant myeloid origin of abnormal cells from the enlarged lymph nodes. This approach may be routinely applied to investigate cases of myeloproliferative neoplasms with unusual clinical manifestations.

For benign lymphadenopathy, extra-pulmonary tuberculosis was the most common etiology besides a nonspecific inflammatory condition in the present study (21 cases). EUS-FNA appeared to be an efficient method for obtaining pathological samples and distinguishing between tuberculosis-induced necrosis and necrosis resulting from malignancy. The diagnostic accuracy in the cohort from the present study was $92.2 \%$; however, the detailed diagnostic criteria for 
extra-pulmonary tuberculosis based on EUS-FNA require further study and documentation $(2,29,30)$.

The present study had certain limitations. It was a retrospective study, and surgical pathology was not available for the majority of patients at the time of diagnosis. However, clinical follow-ups were performed for $\geq 9$ months.

In conclusion, EUS-FNA is associated with high diagnostic accuracy and low complication rates for evaluating cases of mediastinal and intra-abdominal malignant lymphadenopathy. EUS-FNA in combination with FCM as a minimally invasive and highly sensitive tool should be routinely performed to determine lymphoma, in addition to examining enlarged celiac axis lymph nodes in elderly males, who exhibit an increased risk of malignancy based on the data of the present study.

\section{Acknowledgements}

The authors wish to thank all the staff at the Digestive Endoscopy Center of Tongji Hospital (Wuhan, China) for providing the clinical data.

\section{Funding}

No funding was received.

\section{Availability of data and materials}

All data generated or analyzed during this study are included in this published article.

\section{Author's contributions}

JW and BC conceived the study and prepared a draft manuscript. QC and XW assisted with data collection and modification of the manuscript. YW and WH were operational assistants of EUS-FNA and assisted with data collection. All authors contributed to the study design and commented on the manuscript. All authors read and approved the final manuscript.

\section{Ethics approval and consent to participate}

The present study was approved by The Ethics Committee of Tongji Hospital and informed consent was obtained from each patient or their legally authorized representative.

\section{Consent for publication}

Informed consent was obtained from each patient or their legally authorized representative.

\section{Competing interests}

The authors declare that they have no competing interests.

\section{References}

1. Pugh JL, Jhala NC, Eloubeidi MA, Chhieng DC, Eltoum IA Crowe DR, Varadarajulu S and Jhala DN: Diagnosis of deep-seated lymphoma and leukemia by endoscopic ultrasound-guided fine-needle aspiration biopsy. Am J Clin Pathol 125: 703-709, 2006.
2. Puri R, Vilmann P, Sud R, Kumar M, Taneja S, Verma K and Kaushik N: Endoscopic ultrasound-guided fine-needle aspiration cytology in the evaluation of suspected tuberculosis in patients with isolated mediastinal lymphadenopathy. Endoscopy 42: 462-467, 2010.

3. Nakahara O, Yamao K, Bhatia V, Sawaki A, Mizuno N, Takagi T, Shimizu Y, Koshikawa T, Yatabe Y and Baba H: Usefulness of endoscopic ultrasound-guided fine needle aspiration (EUS-FNA) for undiagnosed intra-abdominal lymphadenopathy. J Gastroenterol 44: 562-567, 2009.

4. Hussain T, Salamat A, Farooq MA, Hassan F and Hafeez M: Indications for endoscopic ultrasound and diagnosis on fine-needle aspiration and cytology. J Coll Physicians Surg Pak 19: 223-227, 2009.

5. Larsen SS, Vilmann P, Krasnik K, Dirksen A, Clementsen $P$, Skov BG, Jacobsen GK, Lassen U, Eigtved A, Berthelsen AK, et al: A comparison of endoscopic ultrasound guided biopsy and positron emission tomography with integrated computed tomography in lung cancer staging. Curr Health Sci J 35: 5-12, 2009.

6. Strand DS, Jeffus SK, Sauer BG, Wang AY, Stelow EB and Shami VM: EUS-guided 22-gauge fine-needle aspiration versus core biopsy needle in the evaluation of solid pancreatic neoplasms. Diagn Cytopathol 42: 751-758, 2014.

7. Mehmood S, Loya A and Yusuf MA: Clinical utility of endoscopic ultrasound-guided fine-needle aspiration in the diagnosis of mediastinal and intra-abdominal lymphadenopathy. Acta Cytol 57: 436-442, 2013.

8. Jhala NC, Jhala D, Eltoum I, Vickers SM, Wilcox CM, Chhieng DC and Eloubeidi MA: Endoscopic ultrasound-guided fine-needle aspiration biopsy: A powerful tool to obtain samples from small lesions. Cancer 102: 239-246, 2004.

9. Chen VK and Eloubeidi MA: Endoscopic ultrasound-guided fine needle aspiration is superior to lymph node echofeatures: A prospective evaluation of mediastinal and peri-intestinal lymphadenopathy. Am J Gastroenterol 99: 628-633, 2004.

10. Devereaux BM, Leblanc JK, Yousif E, Kesler K, Brooks J, Mathur P, Sandler A, Chappo J, Lehman GA, Sherman S, et al: Clinical utility of EUS-guided fine-needle aspiration of mediastinal masses in the absence of known pulmonary malignancy. Gastrointest Endosc 56: 397-401, 2002.

11. Sato M, Shirakami G and Fukuda K: Comparison of general anesthesia and monitored anesthesia care in patients undergoing breast cancer surgery using a combination of ultrasound-guided thoracic paravertebral block and local infiltration anesthesia: A retrospective study. J Anesth 30: 244-251, 2016.

12. Meda BA, Buss DH, Woodruff RD, Cappellari JO, Rainer RO, Powell BL and Geisinger KR: Diagnosis and subclassification of primary and recurrent lymphoma. The usefulness and limitations of combined fine-needle aspiration cytomorphology and flow cytometry. Am J Clin Pathol 113: 688-699, 2000.

13. Young NA, Al-Saleem TI, Ehya H and Smith MR: Utilization of fine-needle aspiration cytology and flow cytometry in the diagnosis and subclassification of primary and recurrent lymphoma. Cancer 84: 252-261, 1998.

14. Deans JP and Polyak MJ: FMC7 is an epitope of CD20. Blood 111: 2492, 2493-2494, 2008.

15. Jamil LH, Kashani A, Scimeca D, Ghabril M, Gross SA, Gill KR, Hasan MK, Woodward TA, Wallace MB and Raimondo M: Can endoscopic ultrasound distinguish between mediastinal benign lymph nodes and those involved by sarcoidosis, lymphoma, or metastasis? Dig Dis Sci 59: 2191-2198, 2014.

16. Nguyen TQ, Kalade A, Prasad S, Desmond P, Wright G, Hart D, Conron $M$ and Chen RY: Endoscopic ultrasound guided fine needle aspiration (EUS-FNA) of mediastinal lesions. ANZ J Surg 81: 75-78, 2011

17. ASGE Standards of Practice Committee; Early DS, Acosta RD, Chandrasekhara V, Chathadi KV, Decker GA, Evans JA, Fanelli RD, Fisher DA, Fonkalsrud L, et al: Adverse events associated with EUS and EUS with FNA. Gastrointest Endosc 77: 839-843, 2013.

18. Yasuda I, Tsurumi H, Omar S, Iwashita T, Kojima Y, Yamada T, Sawada M, Takami T, Moriwaki $\mathrm{H}$ and Soehendra N: Endoscopic ultrasound-guided fine-needle aspiration biopsy for lymphadenopathy of unknown origin. Endoscopy 38: 919-924, 2006.

19. Catalano MF, Sivak MJ Jr, Rice T, Gragg LA and Van Dam J: Endosonographic features predictive of lymph node metastasis. Gastrointest Endosc 40: 442-446, 1994. 
20. Kanamori A, Hirooka Y, Itoh A, Hashimoto S, Kawashima H, Hara K, Uchida H, Goto J, Ohmiya N, Niwa Y and Goto H: Usefulness of contrast-enhanced endoscopic ultrasonography in the differentiation between malignant and benign lymphadenopathy. Am J Gastroenterol 101: 45-51, 2006.

21. Song HJ, Kim JO, Eun SH, Cho YD, Jung IS, Cheon YK, Moon JH, Lee JS, Lee MS, Shim CS, et al: Endoscopic ultrasonograpic findings of benign mediastinal and abdominal lymphadenopathy confirmed by EUS-guided fine needle aspiration. Gut Liver 1: 68-73, 2007.

22. De Potter T, Flamen P, Van Cutsem E, Penninckx F, Filez L, Bormans G, Maes A and Mortelmans L: Whole-body PET with FDG for the diagnosis of recurrent gastric cancer. Eur J Nucl Med Mol Imaging 29: 525-529, 2002.

23. Dewitt J, Ghorai S, Kahi C, Leblanc J, McHenry L, Chappo J, Cramer H, McGreevy K, Chriswell M and Sherman S: EUS-FNA of recurrent postoperative extraluminal and metastatic malignancy. Gastrointest Endosc 58: 542-548, 2003.

24. Ribeiro A, Vazquez-Sequeiros E, Wiersema LM, Wang KK, Clain JE and Wiersema MJ: EUS-guided fine-needle aspiration combined with flow cytometry and immunocytochemistry in the diagnosis of lymphoma. Gastrointest Endosc 53: 485-491, 2001.

25. Nunez AL, Jhala NC, Carroll AJ, Mikhail FM, Reddy VV, Xian RR and Jhala DN: Endoscopic ultrasound and endobronchial ultrasound-guided fine-needle aspiration of deep-seated lymphadenopathy: Analysis of 1338 cases. Cytojournal 9: 14, 2012.
26. Kwong YL: Predicting the outcome in non-Hodgkin lymphoma with molecular markers. Br J Haematol 137: 273-287, 2007.

27. Zinzani PL, Colecchia A, Festi D, Magagnoli M, Larocca A, Ascani S, Bendandi M, Orcioni GF, Gherlinzoni F, Albertini $\mathrm{P}$, et al: Ultrasound-guided core-needle biopsy is effective in the initial diagnosis of lymphoma patients. Haematologica 83: 989-992, 1998.

28. Neiman RS, Barcos M, Berard C, Bonner H, Mann R, Rydell RE and Bennett JM: Granulocytic sarcoma: A clinicopathologic study of 61 biopsied cases. Cancer 48: 1426-1437, 1981.

29. Bhandarkar DS, Shah RS, Katara AN, Shankar M, Chandiramani VA and Udwadia TE: Laparoscopic biopsy in patients with abdominal lymphadenopathy. J Minim Access Surg 3: 14-18, 2007.

30. Puri R, Mangla R, Eloubeidi M, Vilmann P, Thandassery R and Sud R: Diagnostic yield of EUS-guided FNA and cytology in suspected tubercular intra-abdominal lymphadenopathy. Gastrointest Endosc 75: 1005-1010, 2012.

This work is licensed under a Creative Commons Attribution-NonCommercial-NoDerivatives 4.0 International (CC BY-NC-ND 4.0) License. 